\title{
Cubic Hermite Collocation Method for Solving Boundary Value Problems with Dirichlet, Neumann, and Robin Conditions
}

\author{
Ishfaq Ahmad Ganaie, ${ }^{1}$ Shelly Arora, ${ }^{2}$ and V. K. Kukreja ${ }^{1}$ \\ ${ }^{1}$ Department of Mathematics, SLIET, Longowal, Punjab 148106, India \\ ${ }^{2}$ Department of Mathematics, Punjabi University, Patiala, Punjab 147002, India
}

Correspondence should be addressed to V. K. Kukreja; vkkukreja@gmail.com

Received 6 August 2013; Revised 15 December 2013; Accepted 29 December 2013; Published 24 February 2014

Academic Editor: Viktor Popov

Copyright (C) 2014 Ishfaq Ahmad Ganaie et al. This is an open access article distributed under the Creative Commons Attribution License, which permits unrestricted use, distribution, and reproduction in any medium, provided the original work is properly cited.

Cubic Hermite collocation method is proposed to solve two point linear and nonlinear boundary value problems subject to Dirichlet, Neumann, and Robin conditions. Using several examples, it is shown that the scheme achieves the order of convergence as four, which is superior to various well known methods like finite difference method, finite volume method, orthogonal collocation method, and polynomial and nonpolynomial splines and B-spline method. Numerical results for both linear and nonlinear cases are presented to demonstrate the effectiveness of the scheme.

\section{Introduction}

Since time immemorial mathematicians are ardently pursuing the solution of linear or nonlinear two point boundary value problems (BVPs) of the type:

$$
\frac{d^{2} y}{d x^{2}}+\alpha_{1}(x) \frac{d y}{d x}+\alpha_{2}(x) y=f(x), \quad x \in[a, b]
$$

subject to Dirichlet, Neumann, and Robin's boundary conditions.

Such BVPs have wide application in astronomy, biology, boundary layer theory, deflection in cables, diffusion process, electromagnetism, heat transfer, and other topics. It is well known that closed form analytical solution of such problems cannot be obtained in many cases; therefore, numerical techniques such as collocation method $[1,2]$, Bspline interpolation [3], Hermite cubic collocation [4-6], finite difference method [7-9], nonlinear shooting method [10], geometric Hermite interpolation [11], quintic B-spline collocation method [12], polynomial and nonpolynomial spline approaches [13-15], quartic spline solution [16], cubic spline collocation method [17], and finite volume element method [18] are frequently used.

In this study, cubic Hermite collocation method (CHCM) involves cubic Hermite basis function to reduce mathematical complexity. Different linear and nonlinear differential equations are solved subject to Dirichlet, Neumann, and Robin boundary conditions using the present method. Moreover, the decoupling technique [4] used to solve elliptic problems with Neumann and Dirichlet conditions is a particular case of present technique. In this paper, linear and nonlinear boundary value problems reported in recent papers $[2,3,7$, $9,13,14]$ are solved using CHCM. It is worth mentioning that $\mathrm{CHCM}$ is giving better results than finite difference method, finite element method, finite volume method, Bspline method, and polynomial and nonpolynomial spline approach with fourth order of convergence.

The paper comprises five sections. Section 1 deals with general introduction of the problem. Section 2 gives brief description of cubic Hermite collocation method. In Section 3, symbolic solution of (1) is presented. Seven numerical examples are discussed in Section 4 and finally overall conclusions are given in Section 5.

\section{Proposed Technique}

In the present method, the domain is divided into finite elements and then orthogonal collocation method with cubic Hermite as basis function is applied within each element. 
The cubic Hermite interpolant of the function $f$ relative to partition $a=x_{1}<x_{2}<\cdots<x_{N+1}=b$ is a function $s$ that satisfies the following:

(1) on each subinterval $\left[x_{k}, x_{k+1}\right], s$ coincides with a cubic polynomial $s_{k}(x)$,

(2) $s$ interpolates $f$ and $f^{(1)}$ at $x_{1}, x_{2}, \ldots, x_{N+1}$,

(3) $s$ and $s^{(1)}$ are continuous on $[a, b]$.

The cubic Hermite interpolant of $f$ and its first derivative at $x=x_{k}$ requires that

$$
s_{k}\left(x_{k}\right)=f\left(x_{k}\right), \quad s_{k}^{(1)}\left(x_{k}\right)=f^{(1)}\left(x_{k}\right) .
$$

Combining the continuity of $s$ and $s^{(1)}$ at $x=x_{k+1}$ with interpolation of $f$ and its first derivative at $x=x_{k+1}$, one gets

$$
\begin{gathered}
s_{k}\left(x_{k+1}\right)=s_{k+1}\left(x_{k+1}\right)=f\left(x_{k+1}\right), \\
s_{k}^{(1)}\left(x_{k+1}\right)=s_{k+1}^{(1)}\left(x_{k+1}\right)=f^{(1)}\left(x_{k+1}\right) .
\end{gathered}
$$

Hence, $s_{k}(x)$ is a third degree polynomial that interpolates both $f$ and $f^{(1)}$ at $x=x_{k}$ and at $x=x_{k+1}$. Therefore, $s_{k}(x)$ can be written as

$$
\begin{aligned}
s_{k}(x)= & H_{1}^{k}(x) f\left(x_{k}\right)+H_{2}^{k}(x) f^{(1)}\left(x_{k}\right) \\
& +H_{3}^{k}(x) f^{(1)}\left(x_{k+1}\right)+H_{4}^{k}(x) f\left(x_{k+1}\right),
\end{aligned}
$$

where

$$
\begin{aligned}
& H_{2 p-1}^{k}(x) \\
& = \begin{cases}\left(\frac{x-x_{k-1}}{h_{k-1}}\right)^{2}\left(3-\frac{2\left(x-x_{k-1}\right)}{h_{k-1}}\right) ; & x \in\left[x_{k-1}, x_{k}\right] \\
\left(1-\frac{x-x_{k}}{h_{k}}\right)^{2}\left(1-\frac{2\left(x-x_{k}\right)}{h_{k}}\right) ; & x \in\left[x_{k}, x_{k+1}\right] \\
0 ; & \text { otherwise, }\end{cases} \\
& H_{2 p}^{k}(x) \\
& = \begin{cases}-h_{k-1}\left(\frac{x-x_{k-1}}{h_{k-1}}\right)^{2}\left(1-\frac{x-x_{k-1}}{h_{k-1}}\right) ; & x \in\left[x_{k-1}, x_{k}\right] \\
h_{j}\left(1-\frac{x-x_{k}}{h_{k}}\right)^{2}\left(\frac{x-x_{k}}{h_{k}}\right) ; & x \in\left[x_{k}, x_{k+1}\right] \\
0 ; & \text { otherwise. }\end{cases}
\end{aligned}
$$

Here, $p=1,2$ and $k=1,2, \ldots, N$.

The grid points, $x_{k}$, are often called the "knots" of the piecewise polynomial since they are points where polynomials are "tied together." The Hermite polynomials do not require the subsidiary condition to make first derivative continuous. This fact reduces the number of equations by $(N-1)$, where $N$ is the number of elements.

The global variable $x$ varies in the $k$ th element, where $k=$ $1,2, \ldots, N$. A new variable $u=\left(x-x_{k}\right) / h_{k}$ is introduced in $k$ th element in such a way that as $x$ varies from $x_{k}$ to $x_{k+1}$, $u$ varies from 0 to 1 . Orthogonal collocation is applied on local variable $u$.

Approximation of function $y(u)$ in the $k$ th element is given as [6]

$$
\bar{y}(u)=\sum_{i=1}^{4} a_{i+2 k-2} H_{i}(u) .
$$

To apply the collocation method, one must evaluate the trial function (6) and its derivatives at two internal collocation points $u=u_{j}(j=1,2)$. These are given by

$$
\begin{aligned}
& \bar{y}\left(u_{j}\right)=\sum_{i=1}^{4} a_{i+2 k-2} H_{i}\left(u_{j}\right), \\
& \frac{d \bar{y}}{d u}\left(u_{j}\right)=\frac{1}{h_{k}} \sum_{i=1}^{4} a_{i+2 k-2} A_{j i}, \\
& \frac{d^{2} \bar{y}}{d u^{2}}\left(u_{j}\right)=\frac{1}{h_{k}^{2}} \sum_{i=1}^{4} a_{i+2 k-2} B_{j i},
\end{aligned}
$$

where the Hermite polynomials and their first and second derivatives are defined as

$$
\begin{aligned}
& H_{1}\left(u_{j}\right)=\left(1+2 u_{j}\right)\left(1-u_{j}\right)^{2}, \quad H_{2}\left(u_{j}\right)=u_{j}\left(1-u_{j}\right)^{2} h_{k}, \\
& H_{3}\left(u_{j}\right)=u_{j}^{2}\left(3-2 u_{j}\right), \quad H_{4}\left(u_{j}\right)=u_{j}^{2}\left(u_{j}-1\right) h_{k}, \\
& A_{j 1}\left(u_{j}\right)=6 u_{j}^{2}-6 u_{j}, \quad A_{j 2}\left(u_{j}\right)=\left(1-4 u_{j}+3 u_{j}^{2}\right) h_{k}, \\
& A_{j 3}\left(u_{j}\right)=6 u_{j}-6 u_{j}^{2}, \quad A_{j 4}\left(u_{j}\right)=\left(3 u_{j}^{2}-2 u_{j}\right) h_{k}, \\
& B_{j 1}\left(u_{j}\right)=12 u_{j}-6, \quad B_{j 2}\left(u_{j}\right)=\left(6 u_{j}-4\right) h_{k}, \\
& B_{j 3}\left(u_{j}\right)=6-12 u_{j}, \quad B_{j 4}\left(u_{j}\right)=\left(6 u_{j}-2\right) h_{k},
\end{aligned}
$$

where $u_{j}$ 's are the zeros of shifted orthogonal Legendre polynomial $P_{2}^{(0,0)}(u)$ with $u_{1}=0.2113248654$ and $u_{2}=$ 0.7886751346 , as shown in Figure 1.

\section{Symbolic Solution}

Equation (1) can be discretized using (7) as follows:

$$
\begin{gathered}
\frac{1}{h_{k}^{2}} \sum_{i=1}^{4} a_{i+2 k-2} B_{j i}+\beta_{1}(u) \frac{1}{h_{k}} \sum_{i=1}^{4} a_{i+2 k-2} A_{j i}+\beta_{2}(u) \\
\quad \times \sum_{i=1}^{4} a_{i+2 k-2} H_{j i}=f(u)
\end{gathered}
$$

where, in the 1st element, $a_{i}$ 's vary from 1 to 4 , 2nd element varies from 3 to 6 , and so on. Symbolic solution is given subject to Neumann boundary conditions, for $a=0$ and $b=1$ :

$$
\begin{aligned}
& y^{(1)}(0)=c_{0}, \\
& y^{(1)}(1)=c_{L},
\end{aligned}
$$

where $c_{0}$ and $c_{L}$ are finite real constants. 


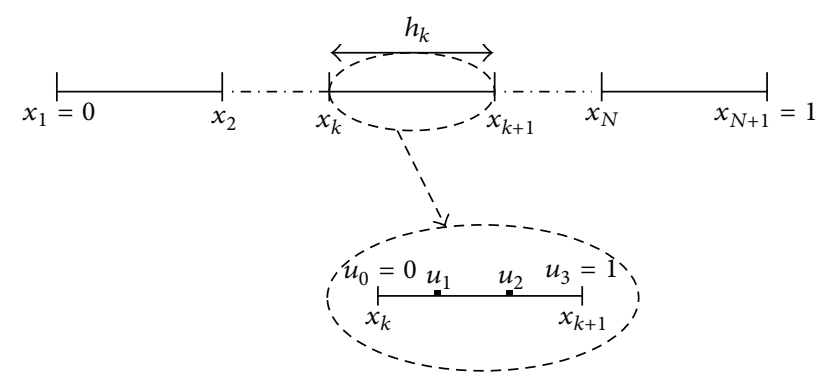

Figure 1: Subdivision of mesh points on the global domain. The four coefficients, in each $k$ element, are estimated by using four collocation points $u_{0}, u_{1}, u_{2}$, and $u_{3}$.

In the first element, $u_{j}=0 \Rightarrow A_{j 1}=A_{j 3}=A_{j 4}=0$; therefore, (10) becomes

$$
\frac{1}{h_{k}} \sum_{i=1}^{4} a_{i} A_{j i}\left(u_{j}\right)=c_{0} \Longrightarrow a_{2}=c_{0} .
$$

In the last element, $u_{j}=1 \Rightarrow A_{j 1}=A_{j 2}=A_{j 3}=0$; therefore, (11) becomes

$$
\frac{1}{h_{k}} \sum_{i=1}^{4} a_{i+2 N-2} A_{j i}\left(u_{j}\right)=c_{L} \Longrightarrow a_{2 N+1}=c_{L} .
$$

A system of $(2 N+2)$ equations is obtained from (9) to (13). It includes all parameters of the system and the dependent variables at the boundaries. The support of each Hermite cubic basis function spans at most two subintervals; therefore, a band matrix is obtained with bandwidth two (Figure 2). Of these, two unknowns are found using boundary conditions and rest $2 N$ are from discretized system of (9), using Mathematica. After substituting the appropriate values of $a$ 's in (7), the result can be obtained for any element.

3.1. Error Analysis. Suppose the function $f(x)$ and its four derivatives are continuous on $[a, b]$ and there is a positive constant which satisfies $\left|f^{4}(x)\right| \leq M$ for all $x \in[a, b]$. If $H(x)$ is the cubic Hermite interpolant of $f$ at $a$ and $b$, then according to de Boor [19], $|f(x)-H(x)| \leq \varepsilon h^{4}$, where $h=$ $b-a, \varepsilon=M / 384$.

Also the placement of the collocation points plays a critical role in obtaining the $O\left(h^{4}\right)$ estimate [20, 21]. For Gauss Legendre roots, as collocation points, an error estimate of $O\left(h^{4}\right)$ is obtained whereas for other choices of the collocation points, only second-order accuracy is obtained.

\subsection{Algorithm of the Method}

Step 1. Divide the domain $0 \leq x \leq 1$ into a mesh $0=x_{1}<$ $x_{2}<\cdots<x_{N+1}=1$.

Step 2. Transform the global variable $x$ into local variable $u=$ $\left(x-x_{k}\right) / h_{k}$.

Step 3. Approximate the solution at $u=u_{j}$.

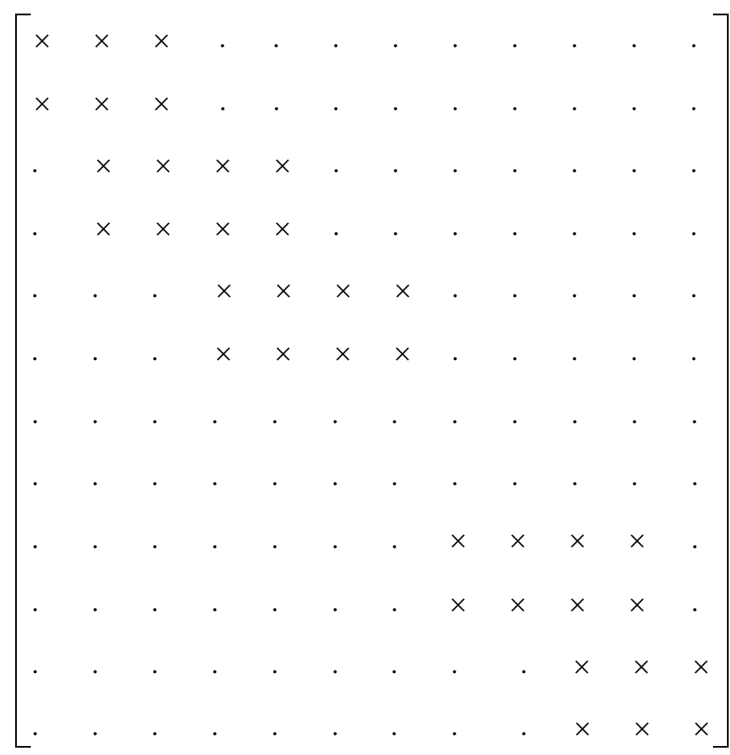

FIGURE 2: Pattern of nonzero elements of banded matrix arising from $\mathrm{CHCM}$ for $N=6$, where $\times$ 's represent nonzero elements and 0 's are represented by dots.

Step 4. Obtain the trial function $\bar{y}\left(u_{j}\right)$ and the derivatives $(d \bar{y} / d u)\left(u_{j}\right),\left(d^{2} \bar{y} / d u^{2}\right)\left(u_{j}\right)$.

Step 5. Carry out discretization of the model using Step 4.

Step 6. Evaluate Step 5 at collocation points $u_{1}=$ 0.2113248654 and $u_{2}=0.7886751346$.

Step 7. The obtained system in Step 6 is solved using any software.

\section{Numerical Examples and Discussion}

In this section, seven examples demonstrate the efficiency and accuracy of the method. Following formulae are used for estimation of error in this study.

Rate of convergence of CHCM is calculated using $\rho=$ $\left(\ln \left(L^{\infty}(N 2) / L^{\infty}(N 1)\right)\right) /(\ln (N 2 / N 1))$.

Relative error is obtained by $\left(y_{\mathrm{ex}}-y_{\mathrm{nm}}\right) / y_{\mathrm{ex}}$.

Max norm is found by $L^{\infty}=\max _{i=1}^{n}\left|y\left(u_{i}\right)-\bar{y}\left(u_{i}\right)\right|$.

Example 1. Solve (1) for

$$
\alpha_{1}(x)=0, \quad \alpha_{2}(x)=-H^{2}, \quad f(x)=0,
$$

subject to Robin's boundary conditions

$$
y(0)=1, \quad y^{(1)}(1)=0,
$$

which has exact solution [9], for planer geometry

$$
y=\frac{\cosh H(1-x)}{\cosh H} \text {. }
$$


TABLE 1: Relative error for different values of parameter $H$ for Example 1.

\begin{tabular}{cccccccc}
\hline & $H=0$ & $H=0.5$ & $H=1$ & $H=2$ & $H=3$ & $H=5$ & $H=10$ \\
& $N=10$ & $N=10$ & $N=10$ & $N=20$ & $N=20$ & $N=30$ \\
\hline 0.0 & 0 & 0 & 0 & 0 & 0 & 0 & 0 \\
0.1 & $1.0000 E-10$ & $5.963 E-11$ & $2.708 E-9$ & $5.1850 E-9$ & $3.6328 E-8$ & $8.9622 E-8$ & $9.0871 E-7$ \\
0.2 & $1.0000 E-10$ & $1.145 E-10$ & $5.337 E-9$ & $1.0504 E-8$ & $7.3297 E-8$ & $1.7941 E-7$ & $1.8174 E-6$ \\
0.3 & $1.0000 E-10$ & $1.640 E-10$ & $7.842 E-9$ & $1.5952 E-8$ & $1.1120 E-7$ & $2.6957 E-7$ & $2.7261 E-6$ \\
0.4 & $1.0000 E-10$ & $2.079 E-10$ & $1.017 E-8$ & $2.1492 E-8$ & $1.5038 E-7$ & $3.6059 E-7$ & $3.6349 E-6$ \\
0.5 & $1.0000 E-10$ & $2.457 E-10$ & $1.229 E-8$ & $2.7024 E-8$ & $1.9106 E-7$ & $4.5343 E-7$ & $4.5440 E-6$ \\
0.6 & $1.0000 E-10$ & $2.773 E-10$ & $1.413 E-8$ & $3.2361 E-8$ & $2.3301 E-7$ & $5.4982 E-7$ & $5.4547 E-6$ \\
0.7 & $1.0000 E-10$ & $3.020 E-10$ & $1.561 E-8$ & $3.7203 E-8$ & $2.7477 E-7$ & $6.5192 E-7$ & $6.3745 E-6$ \\
0.8 & $1.0000 E-10$ & $3.199 E-10$ & $1.673 E-8$ & $4.1147 E-8$ & $3.1262 E-7$ & $7.5863 E-7$ & $7.3351 E-6$ \\
0.9 & $1.0000 E-10$ & $3.307 E-10$ & $1.741 E-8$ & $4.3753 E-8$ & $3.4019 E-7$ & $8.5360 E-7$ & $8.3951 E-6$ \\
1.0 & $1.0000 E-10$ & $3.344 E-10$ & $1.766 E-8$ & $4.4668 E-8$ & $3.5045 E-7$ & $4.2434 E-7$ & $9.0872 E-6$ \\
\hline
\end{tabular}

TABLE 2: Relative error between CHCM and exact values for Example 2.

\begin{tabular}{lccr}
\hline$x$ & Exact solution & CHCM (100 elements) & Relative error \\
\hline 0.0 & 0 & 0 & 0 \\
0.1 & 0.059343034025940 & 0.059343034024546 & $1.3944 E-12$ \\
0.2 & 0.110134207176555 & 0.110134207173893 & $2.6620 E-12$ \\
0.3 & 0.151024408862577 & 0.151024408858821 & $3.7560 E-12$ \\
0.4 & 0.182725813258852 & 0.182725813254164 & $4.6880 E-12$ \\
0.5 & 0.197560538965947 & 0.197560538960736 & $5.2110 E-12$ \\
0.6 & 0.196995306556119 & 0.196995306550776 & $5.3430 E-12$ \\
0.7 & 0.178732867019218 & 0.178732867014236 & $4.9820 E-12$ \\
0.8 & 0.145015397537614 & 0.145015397533471 & $4.1430 E-12$ \\
0.9 & 0.085646323767636 & 0.085646323765122 & $2.5130 E-12$ \\
1.0 & 0 & 0 & 0 \\
\hline
\end{tabular}

TABLE 3: Max norm of errors for five methods with respect to exact solution.

\begin{tabular}{lcc}
\hline Methods & $h$ & Max norm $/ h^{2}$ \\
\hline \multirow{2}{*}{ FDM } & 0.1 & $8.24 E-3$ \\
& 0.01 & $8.31 E-3$ \\
\hline \multirow{2}{*}{ FEM } & 0.1 & $6.35 E-3$ \\
& 0.01 & $6.36 E-3$ \\
\hline \multirow{2}{*}{ FVM } & 0.1 & $3.18 E-3$ \\
& 0.01 & $3.18 E-3$ \\
\hline \multirow{2}{*}{ B-spline } & 0.1 & $2.9 E-4$ \\
& 0.01 & $2.89 E-6$ \\
\hline \multirow{2}{*}{ CHCM } & 0.1 & $7.240 E-4$ \\
& 0.01 & $5.352 E-8$ \\
\hline
\end{tabular}

The problem is solved for different values of dimensionless parameter $H$ by taking 10 to 40 elements. The exact and numeric results are plotted in Figure 3. The results reported by [9] for 10 elements using finite difference method are matching with exact solution up to 3 decimal places, whereas using CHCM the results are matching up to 9 decimal places. This shows the superiority of cubic Hermite collocation

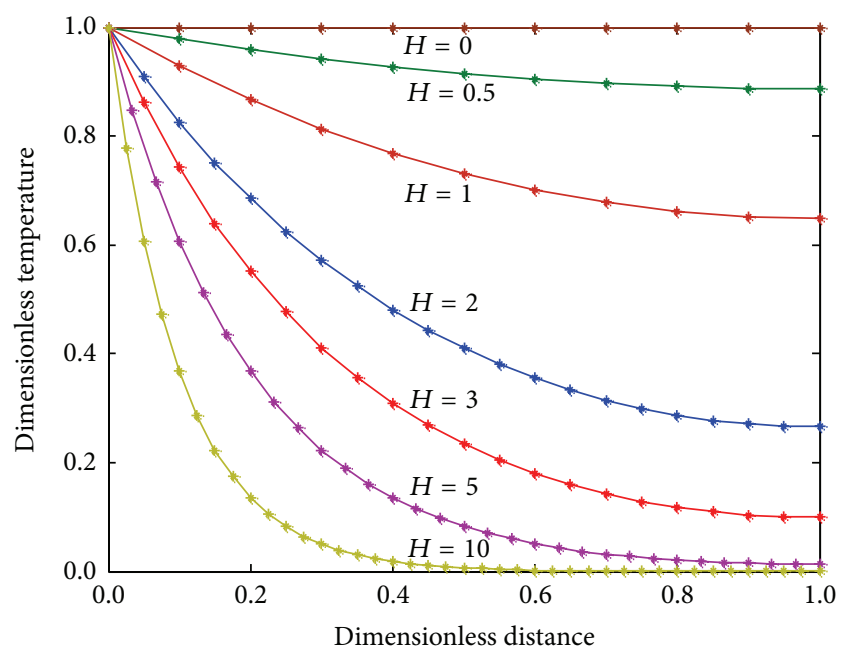

FIgURE 3: Temperature profiles in a rectangular fin for dimensionless heat transfer coefficient $H$.

method over the finite difference method. Relative error between CHCM and exact values is presented in Table 1. 
TABLE 4: Relative error between CHCM and exact values for Example 3.

\begin{tabular}{lccc}
\hline$x$ & Exact solution & CHCM (60 elements) & Relative error \\
\hline 0.1 & 0.100042548871756 & 0.100042549016137 & $1.4400 E-10$ \\
0.2 & 0.110172999814179 & 0.110173000081150 & $2.6700 E-10$ \\
0.3 & 0.131412868154409 & 0.131412868530861 & $3.7600 E-10$ \\
0.4 & 0.165903899721862 & 0.165903900198008 & $4.7600 E-10$ \\
0.5 & 0.217124036179286 & 0.217124036742860 & $5.6400 E-10$ \\
0.6 & 0.290238117151979 & 0.290238117780922 & $6.2900 E-10$ \\
0.7 & 0.392618682595762 & 0.392618683248041 & $6.5200 E-10$ \\
0.8 & 0.534589391091750 & 0.534589391690484 & $5.9900 E-10$ \\
0.9 & 0.730466017481367 & 0.730466017892698 & $4.1100 E-10$ \\
1.0 & 1 & 1 & 0 \\
\hline
\end{tabular}

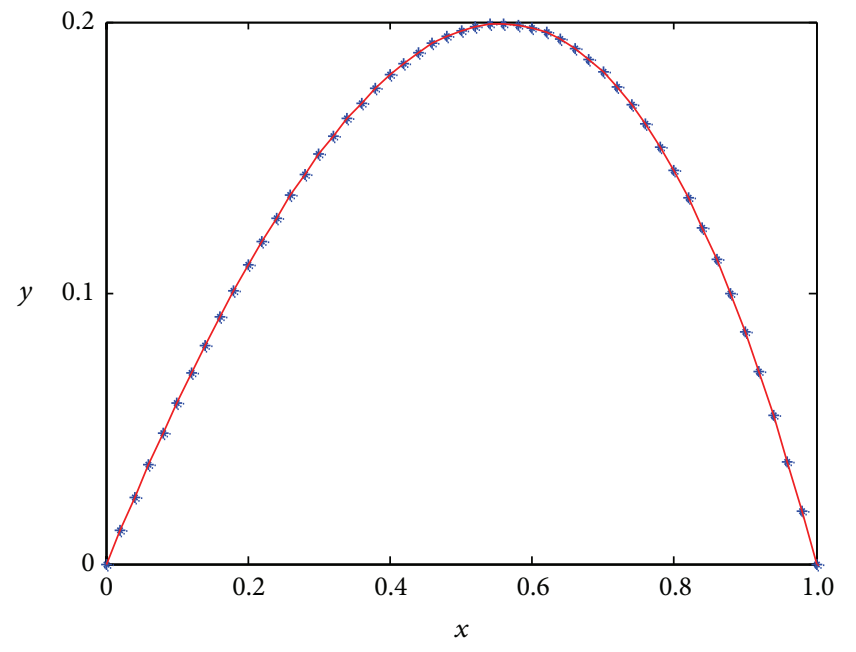

* $\mathrm{CHCM}$

- Exact

FIgURE 4: Comparison of CHCM with exact result for Example 2.

Example 2. Solve (1) for

$$
\alpha_{1}(x)=-1, \quad \alpha_{2}(x)=0, \quad f(x)=-e^{x-1}-1,
$$

subject to Dirichlet boundary conditions

$$
y(0)=y(1)=0, \quad 0<x<1,
$$

which has exact solution $[3,7]$

$$
y(x)=x\left(1-e^{x-1}\right) .
$$

The exact and numeric results are plotted in Figure 4. The CHCM results are matching up to 11 decimal places with the exact ones in Table 2. On comparing present results with the results of $[3,7]$, shown in Table 3 , a big difference of errors between CHCM with finite difference method, finite element method, finite volume method, and B-spline method is observed. This indicates the supremacy of the present method.
TABLE 5: Rate of convergence of CHCM for Example 3.

\begin{tabular}{lcc}
\hline$N_{k}$ & $E\left(N_{k}\right)$ & $\rho$ \\
\hline 0.0666667 & $1.6720 E-07$ & - \\
0.0333333 & $1.0447 E-08$ & 4.000345 \\
0.0166667 & $6.8968 E-10$ & 3.921095 \\
\hline
\end{tabular}

Example 3. Solve (1) for

$$
\alpha_{1}(x)=0, \quad \alpha_{2}(x)=-\phi^{2}, \quad f(x)=0,
$$

subject to Dirichlet boundary conditions

$$
y(0)=0.1, \quad y(1)=1,
$$

which has exact solution [2], for $\phi^{2}=10$ and

$$
y=\frac{g \sinh \phi x+f \sinh \phi(1-x)}{\sinh \phi} .
$$

The exact and numeric results are plotted in Figure 5. The results are matching up to 9 decimal places as shown in Table 4. From Table 5, order of convergence is found to be 4 .

Example 4. Solve (1) for

$$
\alpha_{1}(x)=0, \quad \alpha_{2}(x)=1, \quad f(x)=-1,
$$

subject to Neumann boundary conditions

$$
y^{(1)}(0)=\frac{1-\cos (1)}{\sin (1)}, \quad y^{(1)}(1)=-\frac{1-\cos (1)}{\sin (1)},
$$

which has exact solution $[13,14]$ as

$$
y(x)=\cos x+\frac{1-\cos (1)}{\sin (1)} \sin x-1 .
$$

Example 5. Solve (1) for

$$
\begin{gathered}
\alpha_{1}(x)=0, \quad \alpha_{2}(x)=x \\
f(x)=\left(3-x-x^{2}+x^{3}\right) \sin x+4 \cos x
\end{gathered}
$$


TABLE 6: Maximum absolute errors obtained by different methods in Example 4.

\begin{tabular}{|c|c|c|c|c|c|}
\hline Methods & $N=8$ & $N=16$ & $N=32$ & $N=64$ & $N=128$ \\
\hline Quadratic spline method [14] & $7.70 E-04$ & $1.93 E-04$ & $4.83 E-05$ & $1.21 E-05$ & $3.02 E-06$ \\
\hline Cubic spline method [14] & $7.13 E-04$ & $1.78 E-04$ & $4.45 E-05$ & $1.11 E-05$ & $2.78 E-06$ \\
\hline Nonpolynomial spline method [14] & $1.75 E-04$ & $2.16 E-05$ & $2.68 E-06$ & $3.33 E-07$ & $4.15 E-08$ \\
\hline Polynomial spline approach [13] & $2.68 E-05$ & $4.53 E-07$ & $8.42 E-09$ & $2.21 E-10$ & $6.41 E-12$ \\
\hline Present approach & $1.53 E-07$ & $9.35 E-09$ & $6.11 E-10$ & $3.00 E-11$ & $9.99 E-13$ \\
\hline
\end{tabular}

TABLE 7: Maximum absolute errors obtained by different methods in Example 5.

\begin{tabular}{lccccc}
\hline Methods & $N=8$ & $N=16$ & $N=32$ & $N=64$ & $N=128$ \\
\hline Quadratic spline method [14] & $4.50 E-02$ & $3.08 E-03$ & $7.70 E-04$ & $1.93 E-04$ & $4.80 E-05$ \\
Cubic spline method [14] & $1.15 E-02$ & $2.88 E-03$ & $7.21 E-04$ & $1.80 E-04$ & $4.50 E-05$ \\
Nonpolynomial spline method [14] & $2.67 E-03$ & $3.24 E-04$ & $3.99 E-05$ & $4.94 E-06$ & $6.16 E-07$ \\
Polynomial spline approach [13] & $2.22 E-04$ & $5.05 E-06$ & $1.63 E-07$ & $5.58 E-09$ & $1.89 E-10$ \\
Present approach & $2.34 E-06$ & $1.43 E-07$ & $9.99 E-09$ & $8.00 E-10$ & $6.00 E-11$ \\
\hline
\end{tabular}

TABLE 8: Rate of convergence of CHCM for Examples 4 and 5.

\begin{tabular}{lcccc}
\hline \multirow{2}{*}{$N_{k}$} & \multicolumn{2}{c}{ Example 4} & \multicolumn{2}{c}{ Example 5 } \\
& $E\left(N_{k}\right)$ & $\rho$ & $E\left(N_{k}\right)$ & $\rho$ \\
\hline $1 / 8$ & $1.53 E-07$ & - & $2.34 E-06$ & - \\
$1 / 16$ & $9.35 E-09$ & 4.03 & $1.43 E-07$ & 4.04 \\
$1 / 32$ & $6.11 E-10$ & 3.93 & $9.99 E-09$ & 3.84 \\
$1 / 64$ & $3.00 E-11$ & 4.35 & $8.00 E-10$ & 3.64 \\
\hline
\end{tabular}

TABLE 9: Rate of convergence of CHCM for Examples 6 and 7.

\begin{tabular}{lcccc}
\hline \multirow{2}{*}{$N_{k}$} & Example 6 & \multicolumn{2}{c}{ Example 7 } \\
& $E\left(N_{k}\right)$ & $\rho$ & $E\left(N_{k}\right)$ & $\rho$ \\
\hline $1 / 25$ & $2.4810 E-03$ & - & $3.3211 E-03$ & - \\
$1 / 50$ & $1.8600 E-04$ & 3.74 & $1.9900 E-04$ & 4.07 \\
$1 / 100$ & $1.1000 E-05$ & 4.08 & $1.2000 E-05$ & 4.06 \\
\hline
\end{tabular}

subject to Neumann boundary conditions

$$
y^{(1)}(0)=-1, \quad y^{(1)}(1)=2 \sin (1),
$$

which has exact solution $[13,14]$ as

$$
y(x)=\left(x^{2}-1\right) \sin x
$$

For different number of elements $N=8,16,32,64$, and 128 , Examples 4 and 5 are solved using the present method. Maximum absolute errors of the numerical solutions are calculated and compared with those reported by $[13,14]$, for different methods, in Tables 6 and 7. The present approach is giving much more accurate results than the others. From Table 8, the order of convergence is found to be 4 .

Example 6. Consider a nonlinear problem, that is, solving (1) for

$$
\begin{gathered}
\alpha_{1}(x)=0, \quad \alpha_{2}(x)=-y, \\
f(x)=2 \pi^{2} \cos (2 \pi x)-\sin ^{4}(\pi x),
\end{gathered}
$$

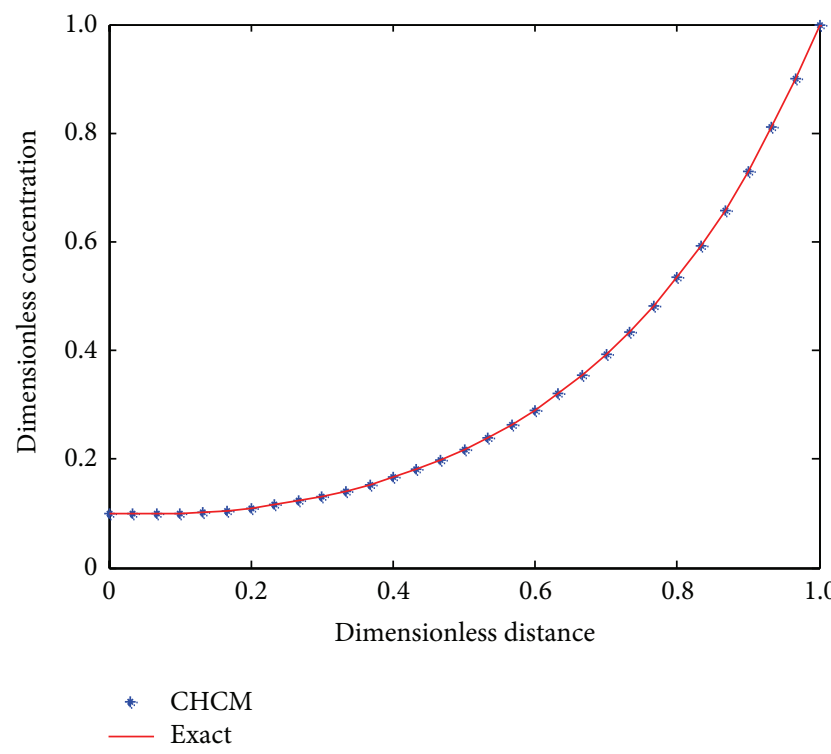

FIGURE 5: Comparison of CHCM with exact result for Example 3.

subject to Neumann boundary conditions

$$
y^{(1)}(0)=0, \quad y^{(1)}(1)=0,
$$

which has exact solution [13]

$$
y(x)=\sin ^{2}(\pi x) .
$$

The exact and CHCM results are showing good agreement in Figure 6 for $N=50$.

Example 7. Consider a nonlinear problem, that is, solving (1) for

$$
\alpha_{1}(x)=0, \quad \alpha_{2}(x)=\frac{e^{-2 y}}{y}, \quad f(x)=0,
$$




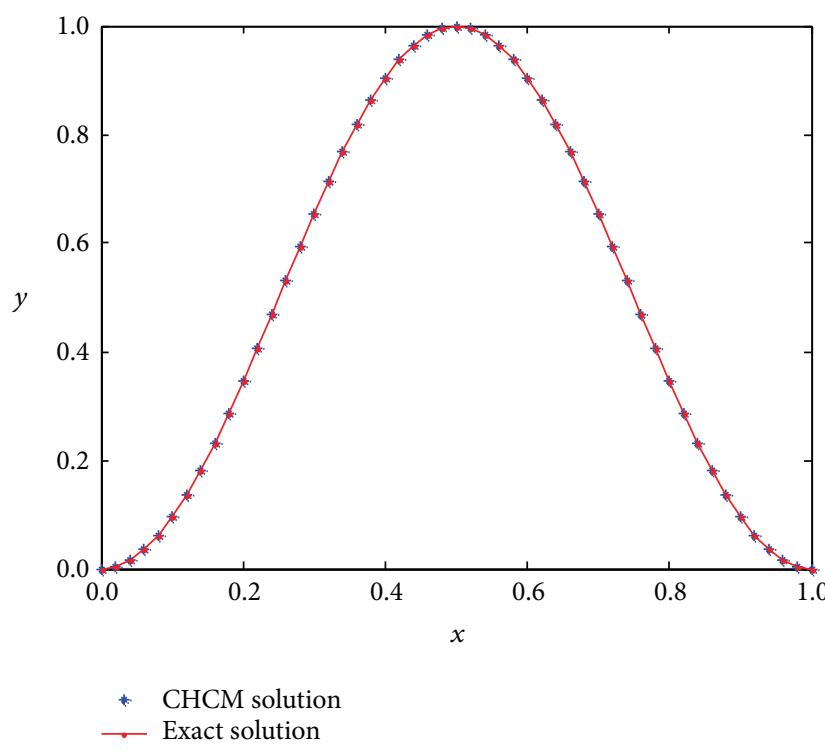

Figure 6: Comparison of CHCM with exact result for Example 6.

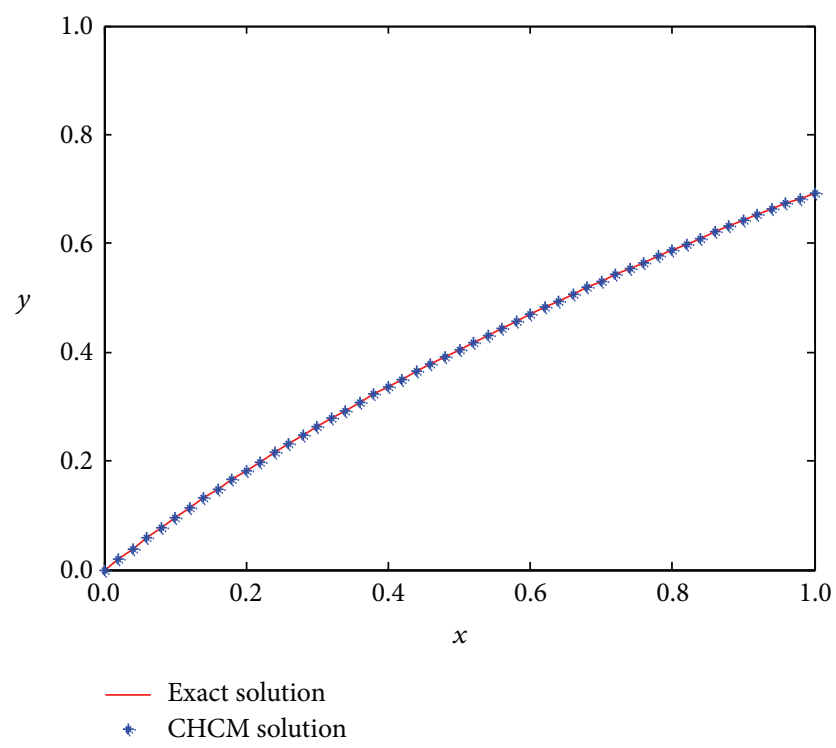

FIgURE 7: Comparison of CHCM with exact result for Example 7.

subject to Neumann boundary conditions

$$
y^{(1)}(0)=1, \quad y^{(1)}(1)=\frac{1}{2},
$$

which has exact solution [13]

$$
y(x)=\ln (1+x) .
$$

An excellent matching is found between the exact and CHCM results in Figure 7 for $N=50$. In Table 9, the order of convergence is again found to be 4 for Examples 6 and 7 .

\section{Conclusion}

In this paper, cubic Hermite collocation method is tested for seven problems. The numerical results obtained are quite satisfactory and comparable with the existing solution available in the literature. The superiority over the finite difference method, finite element method, finite volume method, Bspline method, and polynomial and nonpolynomial spline approach shows the strength of this method. The convergence of the CHCM technique is of order 4.

\section{Conflict of Interests}

The authors declare that there is no conflict of interests regarding the publication of this paper.

\section{Acknowledgments}

This work is supported by NBHM, Mumbai, and UGC, New Delhi, India, in the form of Research Projects 2/48(14)/2009/ R\&D-II/2806 and 41-786/2012(SR), respectively. The JRF provided to Mr. I. A. Ganaie by NBHM, Mumbai, is thankfully acknowledged.

\section{References}

[1] J. H. Ahlberg and T. Ito, "A collocation method for two point boundary value problems," Mathematics of Computation, vol. 29, no. 131, pp. 761-776, 1975.

[2] M. A. Soliman and A. A. Ibrahim, "Studies on the method of orthogonal collocation III: the use of Jacobi orthogonal polynomials for the solution of boundary value problems," Journal of King Saud University, vol. 11, no. 2, pp. 191-202, 1999.

[3] H. Caglar, N. Caglar, and K. Elfaituri, "B-spline interpolation compared with finite difference, finite element and finite volume methods which applied to two-point boundary value problems," Applied Mathematics and Computation, vol. 175, no. 1, pp. 72-79, 2006.

[4] W. R. Dyksen and R. E. Lynch, "A new decoupling technique for the Hermite cubic collocation equations arising from boundary value problems," Mathematics and Computers in Simulation, vol. 54, no. 4-5, pp. 359-372, 2000.

[5] D. J. Higham, "Monotonic piecewise cubic interpolation, with applications to ODE plotting," Journal of Computational and Applied Mathematics, vol. 39, no. 3, pp. 287-294, 1992.

[6] P. M. Prenter, Splines and Variational Methods, Wiley interscience, New York, NY, USA, 1975.

[7] Q. Fang, T. Tsuchiya, and T. Yamamoto, "Finite difference, finite element and finite volume methods applied to two-point boundary value problems," Journal of Computational and Applied Mathematics, vol. 139, no. 1, pp. 9-19, 2002.

[8] M. Kumar, "A second order finite difference method and its convergence for a class of singular two-point boundary value problems," Applied Mathematics and Computation, vol. 146, no. 2-3, pp. 873-878, 2003.

[9] V. R. Subramanian and R. E. White, "Symbolic solutions for boundary value problems using Maple," Computers and Chemical Engineering, vol. 24, no. 11, pp. 2405-2416, 2000. 
[10] S. N. Ha, "A nonlinear shooting method for two-point boundary value problems," Computers and Mathematics with Applications, vol. 42, no. 10-11, pp. 1411-1420, 2001.

[11] M. Krajnc, "Geometric Hermite interpolation by cubic G1 splines," Nonlinear Analysis: Theory, Methods and Applications, vol. 70, no. 7, pp. 2614-2626, 2009.

[12] F.-G. Lang and X.-P. Xu, "Quintic B-spline collocation method for second order mixed boundary value problem," Computer Physics Communications, vol. 183, no. 4, pp. 913-921, 2012.

[13] L.-B. Liu, H.-W. Liu, and Y. Chen, "Polynomial spline approach for solving second-order boundary-value problems with Neumann conditions," Applied Mathematics and Computation, vol. 217, no. 16, pp. 6872-6882, 2011.

[14] M. A. Ramadan, I. F. Lashien, and W. K. Zahra, "Polynomial and nonpolynomial spline approaches to the numerical solution of second order boundary value problems," Applied Mathematics and Computation, vol. 184, no. 2, pp. 476-484, 2007.

[15] S. S. Siddiqi and G. Akram, "Solution of fifth order boundary value problems using nonpolynomial spline technique," Applied Mathematics and Computation, vol. 175, no. 2, pp. 1574-1581, 2006.

[16] S. S. Siddiqi, G. Akram, and A. Elahi, "Quartic spline solution of linear fifth order boundary value problems," Applied Mathematics and Computation, vol. 196, no. 1, pp. 214-220, 2008.

[17] W. Sun, "Hermite cubic spline collocation methods with upwind features," ANZIAM Journal, vol. 42, pp. 1379-1397, 2000.

[18] Z. Xiong and Y. Chen, "Finite volume element method with interpolated coefficients for two-point boundary value problem of semilinear differential equations," Computer Methods in Applied Mechanics and Engineering, vol. 196, no. 37-40, pp. 37983804, 2007.

[19] C. de Boor, A Practical Guide To Splines, Springer, New York, NY, USA, 2001.

[20] J. Douglas and T. Dupont, "A finite element collocation method for quasi-linear parabolic equations," Mathematics of Computation, vol. 121, pp. 17-28, 1973.

[21] I. A. Ganaie, B. Gupta, N. Parumasur, P. Singh, and V. K. Kukreja, "Asymptotic convergence of cubic Hermite collocation method for axial dispersion model," Applied Mathematics and Computation, vol. 220, no. 1, pp. 560-567, 2013. 


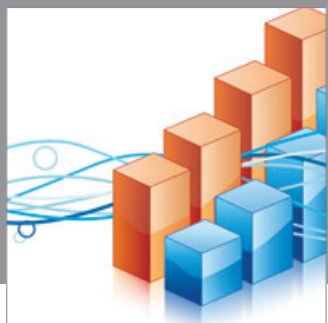

Advances in

Operations Research

mansans

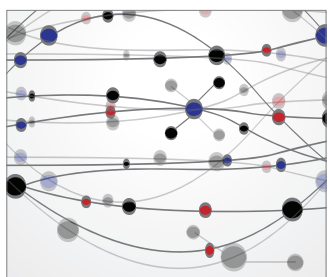

The Scientific World Journal
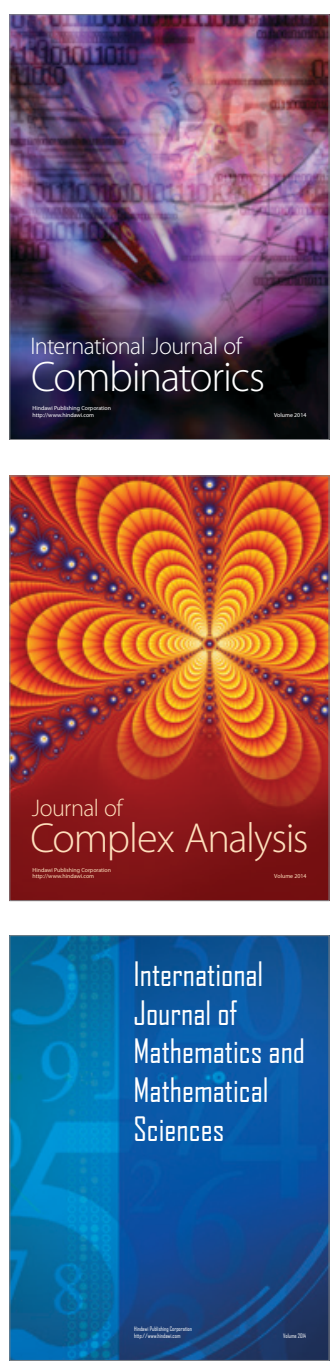
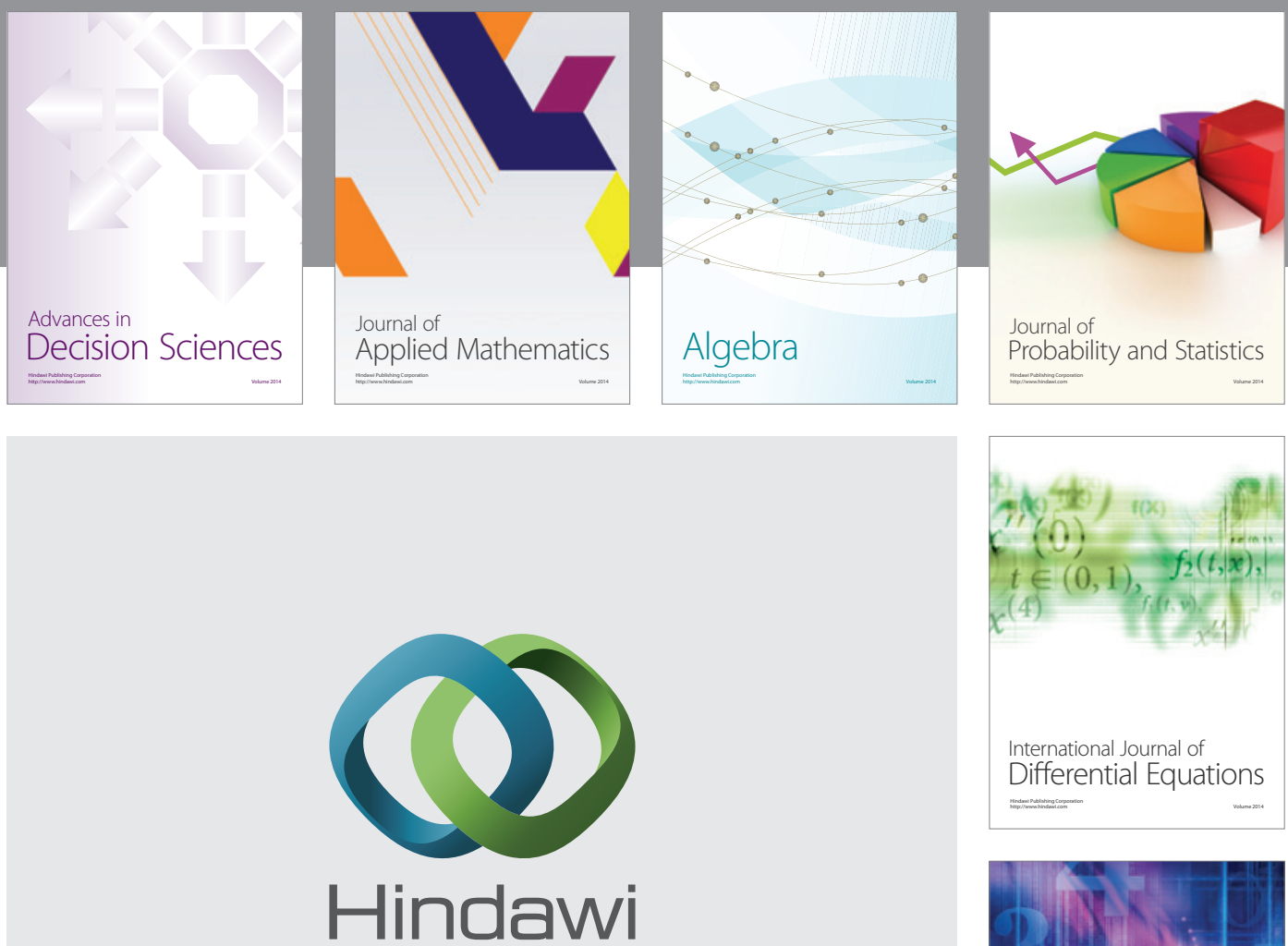

Submit your manuscripts at http://www.hindawi.com
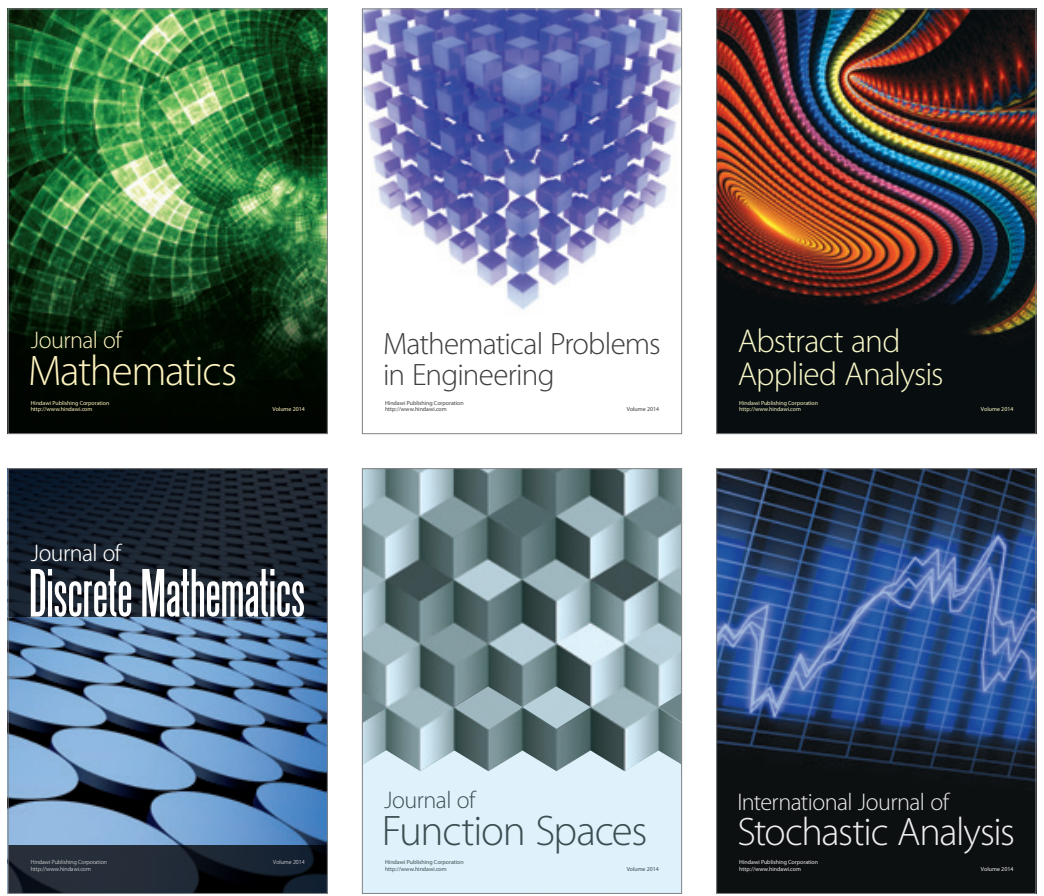

Journal of

Function Spaces

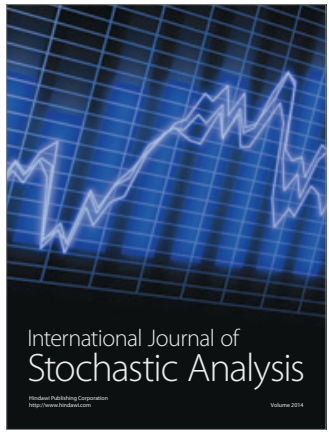

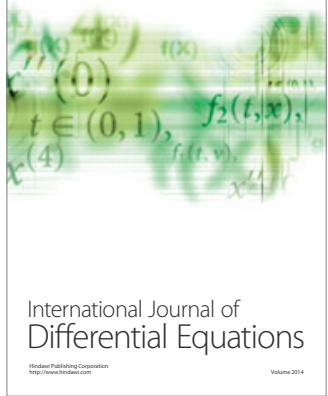
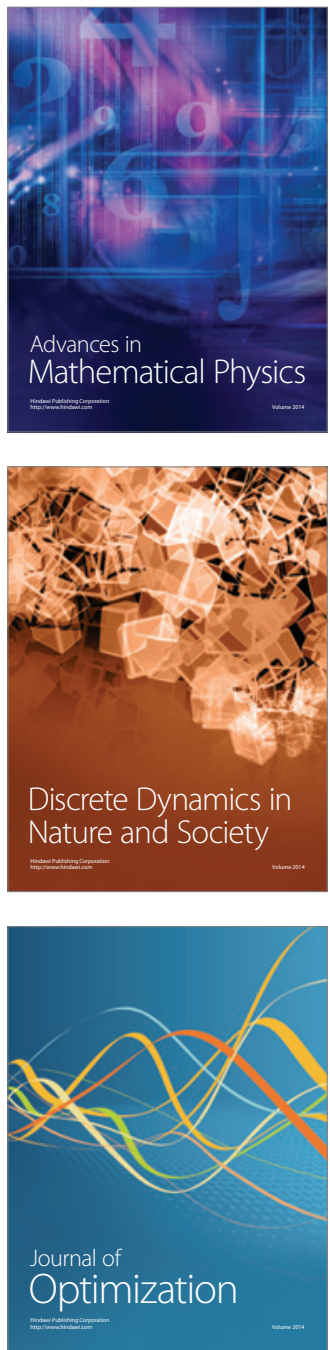